\title{
Integrative multi-omics profiling of resectable pancreatic cancer reveals clinically relevant molecular subtypes with precision strategies beyond the clinical staging system
}

\author{
Sung Hwan LEE, ${ }^{* 1}$, Hye Jung CHO', Incheon KANG', Sung Hoon CHOI', Sunyoung LEE², Ju-Seog LEE \\ 'Department of Surgery, CHA Bundang Medical Center, CHA University School of Medicine, Seongnam, Korea \\ ${ }^{2}$ Department of Gastrointestinal Medical Oncology, University of Texas MD Anderson Cancer Center, Houston, TX, USA \\ ${ }^{3}$ Department of Systems Biology, University of Texas MD Anderson Cancer Center, Houston, TX, USA
}

Introduction: Even though the current clinical staging system for resectable pancreatic cancer has validated major clinicopathologic factors in multiple clinical cohorts, there is still an unmet need for integrative consideration using multi-omics data to stratify the patients with pancreatic cancer elaborately.

Methods: We performed a comprehensive analysis and profiling using genomic, transcriptomic, and proteomic data from TCGA-PAAD ( $\mathrm{n}=150)$ and other translational cohorts (4 cohorts, $\mathrm{n}=340)$. Molecular features and major subtypes were analyzed mutually with clinical and pathologic factors to discover a clinically relevant translational staging system.

Results: The correlation analysis for each 50 cancer-specific molecular pathways with the clinical staging system revealed no remarkable pattern of the association. The mutational pattern of KRAS and several transcriptomic pathways, such as epithelial-mesenchymal transition and DNA repair, were differently presented in each clinical stage from the 8th AJCC TNM staging system. From the cluster of cluster analysis, we identified three consensus molecular subtypes, specifically subtype A was remarkably correlated with previously known aggressive subtypes, Basal, Mesenchymal, and Squamous subtypes, and subtype B and C were correlated with other subtypes regarding classical, exocrine, immunogenic, progenitor and ADEX subtypes. This consensus subtypes were validated at the independent pancreatic cancer genomics cohorts. Our in-silico prediction revealed subtype-specific biomarkers and potential therapeutics, including molecular target agents as well as immunotherapeutic options.

Conclusions: Our comprehensive multi-omics analysis reveals prognostically significant consensus subtypes showing distinct tumor biology with unique therapeutic opportunities including biomarkers with high performance. This study provides exact needs for clinical trials based on translational approaches to establish precision surgical oncology. 